\title{
Optimal Control of Tumor Growth Model with Dendritic Cells as Immunotherapy
}

\author{
Firmansyah Reskal Motulo ${ }^{1}$, Trisilowati $^{2 *}$, Abdul Rouf ${ }^{2}$ \\ ${ }^{1}$ Master Program Mathematics, Faculty of Mathematics and Natural Sciences, University of Brawijaya, Malang, \\ Indonesia \\ ${ }^{2}$ Department of Mathematics, Faculty of Mathematics and Natural Sciences, University of Brawijaya, Malang, Indonesia
}

\begin{abstract}
In this paper, optimal control of tumor growth model with dendritic cells as immunotherapy is provided. The model equation can be expressed into a nonlinear differential equation system consisting of four compartments namely, tumor cells, CTL cells, helper T cells, and dendritic cells. Dendritic cells as immunotherapy are injected to the body at time t. The aim of this optimal control is to minimize the tumor cells density as well as the cost of dendritic cells to be administered to the body. Optimal control problem is carried out based on Pontryagin's maximum principle and numerical simulation is solved by using Forward-Backward Sweep methods. Simulation results show that control strategy shrinks tumor cells density which is shown by tumor cells density graph that monotonically decreases after applying dendritic cells as immunotherapy.
\end{abstract}

Keywords: immunotherapy, optimal control, Tumor cell.

\section{INTRODUCTION}

Cancer is a term that commonly used to address a group of dangerous diseases. One characteristic of cancer as an abnormal cell is uncontrolled growth. Cancer is caused by a malignant tumor, in general tumor is divided into two, namely benign tumors and malignant tumors (cancer). Cancer is formed from abnormal and unstable cells and developed through the bloodstream system and lymphatic system [1]. Cancer arises from the transformation of healthy cells into tumor cells in a multi-stage process that generated from the pre-cancerous stage to malignant tumors. This changes are commonly occurring because genetic factors and three external factors, namely physical carcinogens, such as ultraviolet radiation and ionization; chemical carcinogens such as asbestos, tobacco smoke components, aflatoxins (food's contaminant), and arsenic (drinking water's contaminant); and biological carcinogens such as infections from viruses, bacteria or certain parasites [2].

Based on WHO data in 2010, the number of cancer patients worldwide increases every year, which is firm to 7 million people with two-thirds of them are in developing countries [3]. There are several ways that can be proposed to inhibit

\footnotetext{
* Correspondence address:

Trisilowati

Email : trisilowati@ub.ac.id

Address : Dept. Mathematics, Faculty of Mathematics and Natural Sciences, University of Brawijaya, Veteran Malang, Malang 65145.
}

tumor growth such as radiation and drug therapy. Also, it ultimately can be done through immunotherapy by using dendritic cells (DCs) which are known as dendritic cell vaccinations. A dendritic cell vaccine is a new form of immunotherapy used to treat cancer [4].

Dendritic cells are the most effective antigenpresenting cell because of its strategic location, such in places where microbes and foreign antigens enter the body and are located around the organs that targeted by the bacteria or abnormal cells progression [5]. Based on its function there are two types of dendritic cells, namely conventional dendritic cells (cDCs) and plasmacytoid dendritic cells ( $p D C s$ ). The primary feature of $c D C s$ is to recognize the antigens then presented to $\mathrm{T}$ cells $\left(\mathrm{CD} 4^{+} \mathrm{T}\right)$ and removed some crucial molecules, while pDCs have a role in generating interferon as antiviral in the body. In general, it can be illustrated that the part of dendritic cells against tumor or other infections started by recognizing such antigens produced by the abnormal cell, then releasing molecules such as cytokines to activate $\mathrm{T}$ cells by presenting antigen through the major histocompatibility complex (MHC) or human leucocyte antigen (HLA) [6].

Analysis of tumor growth model has been performed by several researchers, including Kirschner and Panetta that discussed mathematical models of tumor growth and their interactions with immunotherapy [7]. This study described the dynamics between tumor cells, immune cells, and IL-2 which prove that 
immunotherapy with interleukin- 2 can boost the immune system [7].

DePillis et al. in 2001 evaluated a tumorspreading model that expressed in the form of a system of ordinary differential equations. In this study, an optimal control approach is used to evaluate the tumor growth with drug therapy. In this model, there are four subpopulations, namely tumor cells, host cells, immune cells, and drug therapy [8]. Five years later DePillis et al. developed a tumor growth model by combining immunotherapy and chemotherapy. The results of numerical simulations indicated that chemotherapy or immunotherapy alone is not sufficient to control tumor growth, furthermore it is shown that combining the two therapies can significantly remove the entire tumor [9].

In 2013, DePillis et al. analyzed the mathematical model by performing therapy on melanoma using dendritic cells [10]. In that model, they have modified the Ludewig's model describing the interaction between dendritic cells, organs, and tumor cells [11]. In the same year, Trisilowati et al. researched optimal control of dendritic cell treatment model on tumor growth. It is demonstrated that dendritic cells are used as a natural killer for tumor cells and treatment therapy which is solved using optimal control problems [12].

Sharma and Samanta in 2013 conducted a study on tumor growth models and their interactions with the immune system using chemotherapy drugs [13]. The analysis of tumor growth model is performed by investigating the effects of tumor immune interactions and chemotherapy drugs which are expressed in four components: (i) T cells that can not attack and destroy tumor cells directly, but release the interleukin-2 cytokine that stimulates CTLS (Cytotoxic T-Lymphocytes) and convert it into active CTL cells, (ii) active CTL cells that can attack, destroy, or even swallow tumor cells, (iii) tumor cells, and (iv) chemotherapy drugs [13]. Sharma and Samanta also applied the model in optimal control problem in order to minimize the amount of drugs to be used in reducing the number of tumor growth. Furthermore, in 2017, Rangel-Reyes et al. conducted an optimal control study of the murine model to measure the effectiveness of dendritic cells [14]. The model consists of tumor cells, $\mathrm{CD} 4^{+} \mathrm{T}$ cells as helper cells, CD8 ${ }^{+} \mathrm{T}$ or CTL cells, dendritic cells, IL-2, TGF- $\beta^{+} \mathrm{T}$ as inhibitory cells, IFN- $\gamma$ which increases regulation of MHC class 1, and the number of MHC class 1 for each melanoma cell [14].
As described in previous study, dendritic cell has an important role in regulating and controlling the tumor growth $[15,16]$. In addition, dendritic cell has a potential application in cancer treatment. This treatment is often considered as a safe treatment compared with other therapies [17]. Therefore, in this research the effect of dendritic cell as immunotherapy in cancer treatment is observed. Mathematical model on Sharma and Shamanta [13] is modified by changing the chemotherapy compartment with dendritic cell as immunotherapy. Optimal control is then applied to the model to find the number of dendritic cells to be administered so as minimize the number of tumor cells as well as the cost of the treatment. Optimal control problems is solved using Pontryagin's maximum principle to obtained the optimal system. The interpretation is performed based on the results of a numerical simulation on MATLAB software by using Sweep Forward-Backward method.

\section{MATERIAL AND METHOD \\ Construction Model}

In this article, optimal control of the tumor growth model consists of four compartments in the form of nonlinear differential equations. Further, each compartment express the density of tumor cells, cell CTLs, helper $\mathrm{T}$ cells, and dendritic cells.

\section{Determination of Optimal Control Problems}

Optimal control settlement is about determining the state, costate, and stationary condition to obtain optimal solutions [18]. In the field of mathematics, optimal control is generally used as a strategy to minimize or maximize a particular objective functional. Therefore, in this paper, the aim of optimal control is to reduce the number of tumor cell as well as the cost of treatment.

\section{Numerical Simulation}

Numerical simulation is conducted to support the analytical results. At this stage, the simulation is approached by using Sweep Forward-Backward method on MATLAB software.

\section{RESULT AND DISCUSSION Model Formulation}

The model in this paper is govern by the ordinary differential equations which consist of four state variables:

(i) $T$, the tumor cells.

(ii) $I_{H}$, the CTL cells. 
(iii) $I_{R}$, the helper T cells.

(iv) $C$, the dendritic cells.

The growth rate of tumor cell is assumed to grow logistically with the terms $r_{1} T\left(1-p_{1} T\right)$. The presence of active CTL cells in the body can eradicate the tumor cells which is represented by a term $-\alpha_{1} T I_{H}$. In addition, to inhibit the development of tumor cells, a dendritic cell vaccination is given by a response of $q_{1}$, thus the rate of tumor cells killed by dendritic cell is denoted by $q_{1} C T$.

The presence of helper T cells serves to stimulate CTLs by releasing an interleukin-2, thus forming an active CTL cell with the terms $\beta I_{H} I_{R}$. Active CTL cells have function to destroy or attack the presence of tumor cells in the body, they become inactive after interacting with tumor cells. Then the density of CTLs cells is reduced by a term $-\mathrm{C}_{2} \mathrm{TI}_{\mathrm{H}}$. In addition, the density of CTL cells decrease because of the decay rate of active CTLs with the terms $-d I_{H^{*}}$

The density of helper T cells is influenced by the growth of such cell that are assumed to grow logistically with the terms $r_{2} I_{R}\left(1-p_{2} I_{R}\right)$. Based on its function, the presence of helper $\mathrm{T}$ cells in the body can not attack and destroy tumor cells directly, but by stimulating CTLs cells into active CTLs cells causing a reduction in helper $\mathrm{T}$ cell density with term $-\beta I_{H} I_{R}$. The presence of dendritic cell vaccination may affect helper $T$ cells resulting in an increase in helper $\mathrm{T}$ cell density with the term $q_{2} C I_{R}$.

Dendritic cells are a type of cell that can be used in the process of inhibiting tumor growth by providing vaccination of these cells to patients with the tumor. To impede the tumor growth, dendritic cell is injected to the body at time $t$ which is denoted by $u(t)$. Furthermore, dendritic cells decay with the rate $\gamma$, so that their density is reduced by a term $-\gamma C$.

Based on the description above, we can obtain equation system for tumor growth model as follows.

$$
\begin{aligned}
& \frac{d T}{d t}=r_{1} T\left(1-p_{1} T\right)-q_{1} C T-\alpha_{1} T I_{H^{i}} \\
& \frac{d I_{H}}{d t}=\beta I_{H^{\prime}} I_{R}-\alpha_{2} T I_{H}-d I_{H^{\prime}} \\
& \frac{d I_{R}}{d t}=r_{2} I_{R}\left(1-p_{2} I_{R}\right)-\beta I_{H} I_{R}+q_{2} C I_{R} . \\
& \frac{d C}{d t}=u-\gamma C .
\end{aligned}
$$

\section{Optimal Control Problems}

The optimal control strategy used in the tumor growth model is a way to inhibit the development of tumor cell density. In this study, optimal control is performed by using dendritic cells as exterminators of tumors, thus in the system of equations (1) variable control with dendritic cells are denoted by $u(t)$. The optimal control problem on the tumor growth model is expressed by the following objective functional

$$
f(u)=\int_{0}^{t_{f}}\left(T+\frac{1}{2} A u^{2}\right) d t
$$

where $A$ represents the weight quantity of controlling dendritic cell vaccination. Next, we determine the optimal solution indicated by $u^{*}$, so that

$f\left(u^{*}\right)=\min \{f(u): u \in U\}$.

given that,

$u=\{0 \leq u \leq 1, t \in[0, T]\}$.

Before determining the optimal solution $\left(u^{*}\right)$, Hamiltonian function is first established to obtain state equations, costate equations, and stationary conditions. The Hamiltonian function is obtained based on the objective functional and constraints on the system (1) which ere expressed in the form

$$
\begin{aligned}
H & =f(t, \vec{x}, u)+\sum_{i=1}^{4} \sigma_{i}(t) g_{i}(t, \vec{x}, u) x \\
& =T+\frac{1}{2} A u^{2}+\sum_{i=1}^{4} \sigma_{i}(t) g_{i}(t, \vec{x}, u) .
\end{aligned}
$$

Given that $T$ is the number of tumor cells to be minimized, $\sigma_{i}$ is costate variable, and $\theta_{i}$ is the right-hand side of the system (1).

$$
\begin{aligned}
H= & T+\frac{1}{2} A u^{2}+\sigma_{1}\left(r_{1} T\left(1-p_{1} T\right)-q_{1} C T-\alpha_{1} T I_{H}\right) \\
& +\sigma_{2}\left(\beta I_{H} I_{R}-\alpha_{2} T I_{H}-d I_{H}\right)+\sigma_{2}\left(r_{2} I_{R}\left(1-p_{2} I_{R}\right)\right. \\
& \left.-\beta I_{H} I_{R}+q_{2} C I_{R}\right)+\sigma_{4}(u-\gamma C) .
\end{aligned}
$$

Furthermore, based on Hamiltonian function then state equation can be obtained as follows.

\section{State Equations}

The state equation of the tumor growth model of the system (1) is derived from Hamiltonian function toward a system of the costate variable (1) with the following steps

$\vec{x}=\frac{\partial H}{\partial \sigma_{i}}=g_{i}(t, \vec{x}, u)$. given that $i=1,2,3,4$ 
Thus, it is obtained

$\frac{d T}{d t}=\frac{\partial H}{\partial \sigma_{1}}=r_{1} T\left(1-p_{1} T\right)-q_{1} C T-\alpha_{1} T I_{H}$

$\frac{d I_{H}}{d t}=\frac{\partial H}{\partial \sigma_{2}}=\beta I_{H} I_{R}-\alpha_{2} T I_{H}-d I_{H}$

$\frac{d I_{R}}{d t}=\frac{\partial H}{\partial \sigma_{3}}=r_{2} I_{R}\left(1-p_{2} I_{R}\right)-\beta I_{H} I_{R}+q_{2} C I_{R}$,

$\frac{d C}{d t}=\frac{\partial H}{\partial \sigma_{4}}=u-\gamma C$.

with initial conditions $T(0) \geq 0, I_{H}(0) \geq 0$, $I_{R}(0) \geq 0, C(0) \geq 0$. Furthermore, the costate equation is obtained based on the Hamilton function and given as in the following sections.

\section{Costate Equations}

The costate equation model of tumor growth with dendritic cells as control is obtained by the completion stage as follows.

$$
\frac{d \sigma_{i}}{d t}=-\frac{\partial H}{\partial x_{i}} \text {, given that } \partial x_{i}=T_{v} I_{H}, I_{R}, C
$$

Thus, we have

$$
\begin{array}{ll}
\frac{d \sigma_{1}}{d t}=-\frac{\partial H}{\partial T}= & -\left(1+r_{1} \sigma_{1}-2 r_{1} p_{1} T \sigma_{1}-\alpha_{1} I_{H} \sigma_{1}\right. \\
& \left.-q_{1} C \sigma_{1}-\alpha_{2} I_{H} \sigma_{2}\right)_{x} \\
\frac{d \sigma_{2}}{d t}=-\frac{\partial H}{\partial I_{H}}= & -\left(-\alpha_{1} T \sigma_{1}+\beta I_{R} \sigma_{2}-\alpha_{2} T \sigma_{2}-d \sigma_{2}\right. \\
\frac{d \sigma_{3}}{d t}=-\frac{\partial H}{\partial I_{R}}= & -\left(\beta I_{R} \sigma_{3}\right)_{x} \sigma_{2}+r_{2} \sigma_{3}-2 r_{2} p_{2} I_{R} \sigma_{3} \\
\frac{d \sigma_{4}}{d t}= & -\frac{\partial H}{\partial C}=-\left(-q_{1} T \sigma_{3}+q_{2} C \sigma_{3}\right)_{x}
\end{array}
$$

with transversality conditions

$$
\sigma_{1}(T)=\sigma_{2}(T)=\sigma_{a}(T)=\sigma_{4}(T)=0 .
$$

\section{Stationer Conditions}

In the optimal control problem of the tumor growth model, the stationary condition is obtained from the partial derivative of Hamiltonian function toward $u$, that is

$$
\frac{\partial H}{\partial u}=0,
$$

Thus, we obtained

$$
\begin{aligned}
\frac{\partial H}{\partial u} & =A u+\sigma_{4 x} \\
A u+\sigma_{4} & =0_{x} \\
A u & =-\sigma_{4 x} \\
u & =-\frac{\sigma_{4}}{A}
\end{aligned}
$$

Given that $0 \leq u \leq 1$, thus we get the equation as follows

$u^{*}= \begin{cases}-\frac{\sigma_{4}}{A}, & \text { if } 0 \leq-\frac{\sigma_{4}}{A} \leq 1 \\ 0, & \text { if }-\frac{\sigma_{4}}{A} \leq 0 \\ 1_{x} & \text { if }-\frac{\sigma_{4}}{A} \geq 1 .\end{cases}$

In compact notation,

$$
u^{*}=\min \left\{\max \left\{0,-\frac{\sigma_{4}}{A}\right\}, 1\right\} .
$$

Furthermore, the optimal system of tumor growth models is obtained by substituting $u^{*}$ on the system of state and costate equation. Then, we obtained

$$
\begin{aligned}
& \frac{d T}{d t}=r_{1} T^{*}\left(1-p_{1} T^{*}\right)-q_{1} C^{*} T^{*}-\alpha_{1} T^{*} I_{H}^{*}, \\
& \frac{d I_{H}}{d t}=\beta I_{H}^{*} I_{R}^{*}-\alpha_{2} T^{*} I_{H}^{*}-d I_{H^{*}}^{*} \\
& \frac{d I_{R}}{d t}=r_{2} I_{R}^{*}\left(1-p_{2} I_{R}^{*}\right)-\beta I_{H}^{*} I_{R}^{*}+q_{2} C^{*} I_{R}, \\
& \frac{d C}{d t}=u-\gamma C^{*} . \\
& \frac{d \sigma_{1}}{d t}=-1-r_{1} \sigma_{1}+2 r_{1} p_{1} T^{*} \sigma_{1}+\alpha_{1} I_{H}^{*} \sigma_{1}+q_{1} C^{*} \sigma_{1} \\
& \frac{d \sigma_{2}}{d t}=\alpha_{1} T^{*} \sigma_{1}-\beta I_{R}^{*} \sigma_{2}+\alpha_{2} T^{*} \sigma_{2}+d \sigma_{2}+\beta I_{R}^{*} \sigma_{3}, \\
& \frac{d \sigma_{2}}{d t}=-\beta I_{H}^{*} \sigma_{2}-r_{2} \sigma_{3}+2 r_{2} p_{2} I_{R}^{*} \sigma_{3}+\beta I_{H}^{*} \sigma_{3}-q_{2} C \sigma_{3}, \\
& \frac{d \sigma_{4}}{d t}=q_{1} T^{*} \sigma_{1}-q_{2} I_{R}^{*} \sigma_{2}+\gamma \sigma_{4}
\end{aligned}
$$

\section{Numerical method and simulations}

In this section, numerical simulations are conducted by using the initial value $\left(T_{v} I_{H}, I_{R}, C\right)=(150,50,75,35)$ and parameter values as in Table 1 . Based on the graph of numerical simulation, we can easily know every alteration that happened to each subpopulation $T_{v}, I_{H^{\prime}} I_{R}, C$ toward time $(t)$.

The graph of the solution in Figure 1. is shown by $u_{\max }=1$, the weight quantity $A=0.5$, and parameter values as presented in Table 1.

Table 1. Paramer values for simulation

$$
\begin{aligned}
& r_{1}=0.0544 \\
& r_{2}=0.009 x \\
& p_{1}=0.002 x \\
& p_{2}=0.0003 \\
& \alpha_{1}=0.0005 \\
& \alpha_{2}=0.00008 \\
& q_{1}=0.0008 \\
& q_{2}=0.0004 \\
& \beta=0.0005 \\
& d=0.0412 \\
& \gamma=0.01
\end{aligned}
$$


Based on the parameter values, it is obtained that the solution graph on the subpopulation of the tumor cells grow to the high number, however it decreases after the immunotherapy is applied. This result suggests that dendritic cells can be used to minimize tumor cell density. Also, the presence of dendritic cells provides a positive effect on the density of helper $T$ cells that serve to stimulate CTL cells, in turn can eradicate the presence of a tumor cell. Increasing the response received by $C T L$ cells are followed by increasing
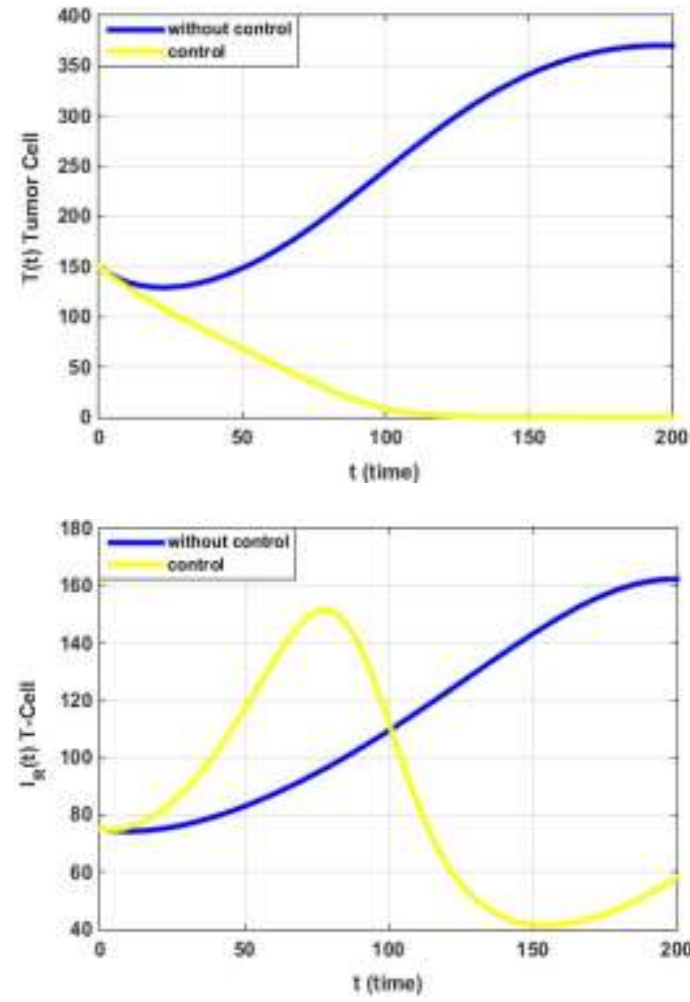

the active CTLs cell density $\left(I_{H}\right)$. Thus, the function of active CTL cells in eradicating the tumor is more optimal (see Fig. 1).

Figure 2 shows the amount of dendritic cell as immunotherapy used in eliminating tumor cells for a given of time. Therapeutic control is provided at maximum value at 1 unit should administered since $t=0$, until it begins to decrease at $t$ ranges from 120 to reach zero when $t$ $=200$. This result states that no more immunotherapy is given.
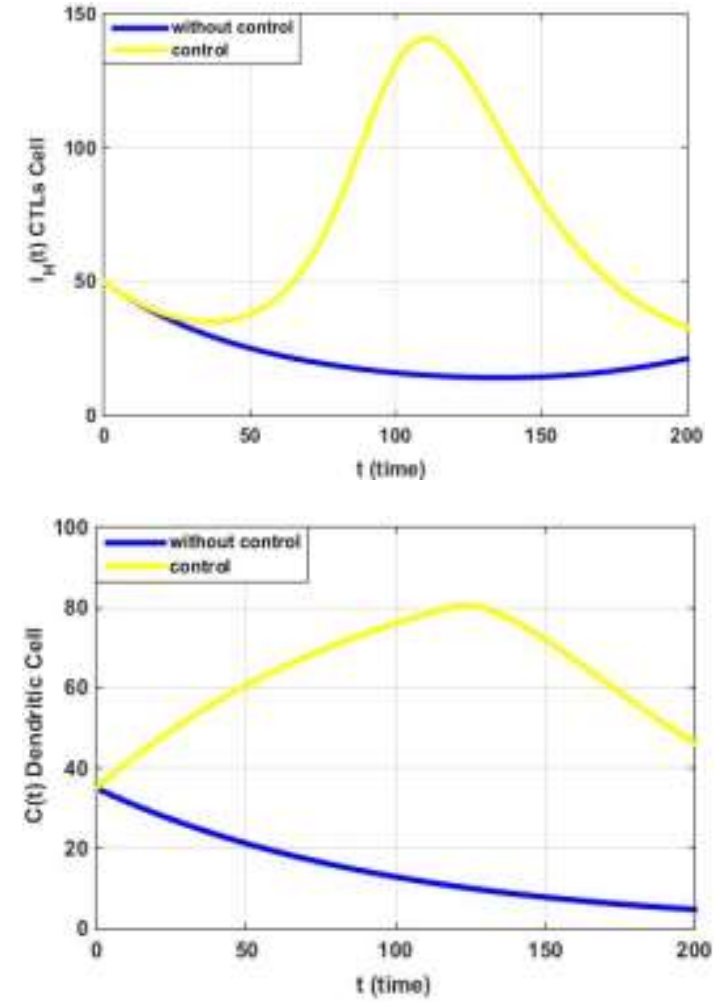

Figure 1. The evolution of subpopulation density $T_{v} I_{H^{x}} I_{R}, C$ for $t \in[0,200]$

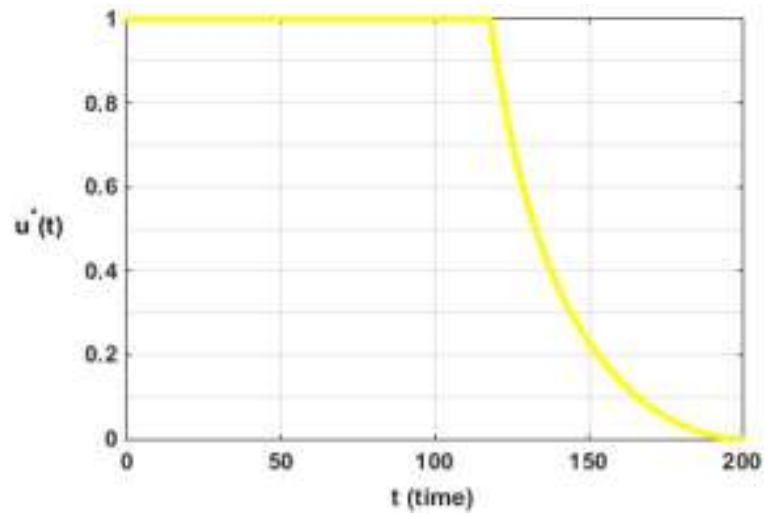

Figure 2. The control profile of immunotherapy, $u(t)$ 


\section{CONCLUSION}

In this paper, we have analyzed an optimal control of tumor growth model with immunotherapy. The effect of dendritic cells as immunotherapy is observed. Optimal system has been derived by using Pontryagin's principle to establish state, costate equation and stationary condition. The results of numerical simulations show that immunotherapy using dendritic cells is effective in reducing the number of tumor cell density. Thus it suggests that occupying dendritic cells as immunotherapy can be used as cancer treatment for the patient in the future.

\section{REFERENCES}

[1] Chalimah, I. N. 2017. Benign tumors and malignant tumors: What is the difference? Available at: http://schema.org/7806/.

[2] World Health Organization. 2017. Cancer. Available at: http://www.who.int/factsheets /fs297/en/.

[3] World Health Organization. 2010. Prevalence of Cancer in Indonesia and Worldwide. Hospital Management, Center for Health Policy and Management. Faculty of Medicine, Gadjah Mada University. Available at: http://www.manajemenrumah sakit.net.

[4] Palucka, K.U., H. Ueno, J. Fay, J. Banchereau. 2010. Dendritic cells and immunity against cancer. J. Internal Med. 269. 64-73.

[5] Bratawijaya, K.G. 2006. Basic immunology, $7^{\text {th }}$ Ed. Medical Faculty, University of Indonesia. Jakarta.

[6] Malissen, B., E. Reinherz, R. Stanfield, I. Wilson. 2012. Chapter 3: the induced responses of innate immunity. In: Murphy, K. (Ed). Janeway's immunobiology $8^{\text {th }}$ Ed. Garland Science Taylor and Paris Group. London and New York. 97-147.

[7] Kirschner, D., J.C. Panetta. 1998. Modeling immunotherapy of the tumor-immune interaction. J. Math. Biol. 37. 235-252.

[8] DePillis, L.G., A.E. Radunskaya. 2001. A mathematical tumor model with immune resistance and drug therapy; an optimal control approach. J. Theor. Med. 3(2). 79100.

[9] DePillis, L.G., W. Gu, A.E. Radunskaya. 2006. Mixed immunotherapy and cemotherapy of tumors; modeling, application and biological interpretation. J. Theor. Biol. 208. 841-862.
[10] DePillis, L.G., A. Gallegos, A.E. Radunskaya. 2013. a model of dendritic cell therapy for melanoma. Front. Oncol. 3. 56.

[11] Ludewig, B.B., P. Krebs, T. Junt, H. Metters, J. Ford, R.M. Anderson. 2004. Deterniming control parameters for Dendritic CellCytotoxic T Lymphocyte interaction. Eur. J. Immunol. 2407-2418.

[12] Trisilowati, S.W. McCue, D.G. Mallet. 2013. An optimal control model of dendritic cell treatment of a growing tumour. ANZIAM Journal. 54, (CTAC 2012), C664-C680.

[13] Sharma, S., G.P. Shamanta. 2013. Dynamical behavior of a tumor-immune system with chemotherapy and optimal control. J. Nonlinear Dyn. 13. 608598.

[14] Rangel-Reyes, J.C., J.C. Chimal-Eguia, E. Castillo-Montiel. 2017. Dendritic immunotherapy improvement for and optimal control murine model. Comput. Math. Methods Med. 9. 5291823.

[15] Munich, S., A. Sobo-Vujanovic, W.J. Buchser et al. 2012. Dendritic cell exesomes directly kill tumor cells and activate natural killer cells via TNF superfamily ligands. Oncolmmunology. 1(7). 1074-1083.

[16] Hanke, N.D. Alizadeh, E. Katsanis, N. Larmonier. 2013. Dendritic cell tumor killing activity and its potential applications in cancer immunotherapy. Crit. Rev. Immunol. 33(1), 1-21.

[17] Sabado, R.L., N. Bhardwaj. 2013. Dendritic cell immunotherapy. Ann. NY Acad. Sci. 1284(1). 31-45.

[18] Lenhart, S., J.T. Workman. 2007. Optimal control applied to biological models. Taylor and Francis Group. New York. 